\title{
Summary of the Third International Symposium on Embryonic Diapause
}

\author{
MB Renfree ${ }^{1}$ and BD Murphy ${ }^{2}$ \\ ${ }^{1}$ School of BioSciences, University of Melbourne, Australia \\ ${ }^{2}$ Centre de recherche en reproduction et fertilité, Université de Montréal, Canada
}

Corresponding author email: $\underline{\text { m.renfree@unimelb.edu.au }}$

\section{Introduction}

What is embryonic diapause? Is it a complete cessation of development or a progression to mitotic silence? Is it merely a slowed development (as occurs in the roe deer and the honey possum)? All of these characteristics fall under the definition of embryonic diapause, while delayed fertilisation or delayed development before the blastocyst stage do not. This Symposium addressed these questions and many others, but the "Big questions" were "Is diapause due to direct inhibition of the blastocyst by the uterus, or is it due to the lack of an essential signal?" and "What are the intracellular signals that are associated with entry of the embryo into diapause and its reactivation from diapause?".

\section{Progress of research on organisms that display diapause}

There were a number of presentations at the Symposium that shed new light on species in taxa known to express this gestational trait. Studies of pandas reported by Wauters et al. (this volume) focussed on the hormonal profiles and food intake that distinguished diapause in pregnant animals from pseudopregnancy. Additional studies by Kailai et al. (abstract only) and Wilson (abstract only) focussed on breeding and urinary steroids in the panda. Similar studies by Bateman and Swanson (abstract only) in two mustelids, the wolverine and the fisher, indicated that the hormone profiles were indistinguishable between diapause and pseudopregnancy. In the mink, it was reported that fecal proteins changed during diapause (Curry et al., this volume). Some novel information on the stoat presented by Amstislavaky et al. (this volume) indicated that newborn females of this species enter estrus within the first month of life, and are directly mated, even before weaning. The consequent embryos enter and remain in diapause until the following spring, a remarkable nine months.

Extensive examination of diapause in the short-tailed fruit bat by Rasweiler and Badwaik (this volume) revealed that developmental arrest of embryos is post-implantational, and at the primitive streak stage, as opposed to the blastocyst stage in many other species known to display embryonic diapause. This condition may more correctly be described as delayed development. This cessation of development in the fruit bat persists for highly variable intervals among individuals and appears to be related to the rainy and dry seasons in their native tropical environment. Investigation of another bat species, the greater short-nosed fruit bat, by Banerjee et al. (abstract only) focused on the role of the adipose depot on developmental arrest and implicated two adipokines, leptin and adiponectin.

Most observational and experimental reports, summarized by Murphy and Fenelon (this volume), have indicated that diapause is a feature of every gestation in pinnipeds. This notwithstanding, new studies, combining ultrasound and observational analyses with molecular studies suggest that the Antarctic species, the Weddell seal, has a very brief, or perhaps non-existent period during 
which the embryo is in diapause (Shero, abstract only). Clearly this needs further examination in this and other pinniped species.

Evaluation of behaviour, growth and fertility comparing offspring of mice from diapause versus non-diapause gestations showed no difference in these traits whether embryonic diapause was present or not during gestation (Zacchini et al., and Fic et al., abstracts only).

\section{New information on the embryo at the beginning of, during and exiting from diapause}

One of the gaps in knowledge addressed by the symposium was how embryos progress into diapause. Fujimori and Kamemizu (abstract only and [1]) cultured mouse embryos in vitro and using live imaging with the transgenic cell cycle marker Fucci2 and Ki67 staining. They showed cell cycle arrest first occurs in the polar trophoblast in the abembryonic trophectoderm regions and spreads to the inner cell mass (ICM) as the mouse blastocyst enters diapause. These studies address the problem of whether cells cease division at the GO or G1 of the cell cycle. The current results suggested that the cell cycle is progressively arrested at the GO phase in later stages of diapause (see also Frankenberg et al. below). One of the striking observations reported confirmed earlier work that the roe deer embryo does undergo continuous, yet largely decelerated development in both the ICM and the trophoblast, with some 15 percent of cells in division prior to the attachment to the uterus (Ruegg et al. and Drews et al. this volume; Ulbrich, abstract only). Mouse embryos were also studied from a molecular perspective by Bulut-Karslioglu (abstract only), who reported that inhibition of mTor in the intercellular growth regulation pathway provoked and maintained diapause. Lim et al. (this volume) reported that the survival of the mouse embryo during diapause requires autophagy, and subsequent recycling of cellular materials. Lipids are a cellular component and energy supply, and morphological data suggest that the depletion of lipids by autophagy characterizes embryonic diapause in the mouse (Ptak et al. this volume; Arena et al., abstract only).

The Symposium was replete with new findings pertinent to the escape from diapause. It was reported that autophagy increases associated with activation, as indicated by an increase in the number of autophagic multivesicular bodies (Lim et al., this volume). This indicated that reactivation is a "last in-first out" process. Global transcriptomic and proteomic analyses of roe deer embryos during the period of decelerated development indicated that extensive remodeling accompanies this phase of embryogenesis, with rapid increase in expression of glycolytic and proliferative genes at activation (van der Weijden et al. this volume and abstract). An extensive report on the reactivation of the embryo from diapause, employing over 10,000 mouse embryos, chronicled the use of transcriptomic and proteomic methods to establish the sequence and significance of the dynamic changes at the cellular level (Kong, this volume). Activation was characterized by the upregulation of a number of signaling molecules including pro-inflammatory signals, suggesting that the reprise of development engenders cellular signalling from the embryo to the endometrium. There was a notable difference between the tammar blastocyst transcriptome between diapause and after reactivation compared to that of the mouse, in that the expression profile of cell cycle genes was not indicative of arrest in G1. Rather, this comparison instead suggested that post-translational regulation of the cell cycle persists until an unusually late stage of development in marsupials (Frankenberg et al., abstract only). Thus, reactivation of the tammar blastocyst from diapause may be tightly linked with the transition from the embryonic to the adult mode of cell cycle regulation. 


\section{Progress in research in cellular biology of the uterus in species with diapause}

In the single report on the morphology of implantation, the mechanisms by which the activated embryo breaches the uterine epithelium were elegantly described (C. Murphy, abstract only). In other presentations, number of identified target genes regulating diapause and consequent reactivation were discussed. Among these was the homeobox factor, Msx, present in the uterus during diapause, and disappearing after reactivation (Cha et al., this volume). Msx is remarkably conserved, as the isoform msx1/MSX1 is found in the mouse and mink uterus, while MSX2 characterizes the wallaby endometrium. The original mouse knock-out study demonstrated that the cytokine leukemia inhibiting factor (LIF) is essential for implantation in the mouse [3]. Further exploration demonstrated that LIF is a conserved factor absent during diapause (Stewart, abstract only; Shaw and Renfree. abstract only), appearing only with reactivation in the wallaby, mink and mouse. A class of polycations known as polyamines, synthesized primarily by the uterus, are now known to be essential players in diapause, in that their absence induces diapause in the mouse and maintains diapause in the mink embryo in vitro (Fenelon et al., this volume). It had previously been shown that the factor that rejuvenates the corpus luteum in this species to terminate diapause is pituitary prolactin. The information presented at the symposium indicated that prolactin has a further role in the mink uterus in provoking the synthesis of polyamines (Fenelon et al. this volume). Other growth factors, including epidermal growth factor (EGF) and heparinbinding EGF-like growth factor (HBEGF) are also conserved factors that are essential for the reactivation of the embryo in all three species above described.

Two studies of the transcriptome of the endometrium related to diapause and reactivation were reported. In the mink, global analyses revealed that more that 350 genes were upregulated during the termination of diapause (Murphy and Fenelon, this volume). Among these were the prolactin receptor and its downstream intracellular signaling molecules and a cluster of genes related to polyamine synthesis. Together, these findings provide further support for the local action of prolactin in terminating diapause. The comparison of the luminal epithelial transcriptome of the roe deer uterus from the time of blastocyst formation until the embryo elongation stage revealed that more than 2500 genes are differentially expressed (van der Weijden et al., this volume and abstract). These changes are reflected in a large-scale increase in uterine fluid secretions containing increasing amounts of hexose, fructose and total proteins (van der Weijden et al., abstract and [4]). Proteomic scrutiny of diapause blastocysts and endometrium is continuing (e.g. [5] [6], Fenelon et al. this volume and Shaw and Renfree (abstract only) but this aspect of global analysis needs to be amplified.

There are a number of studies in recent literature indicating a crosstalk between the preimplantation embryo and the uterus in mammals in the form of extracellular vesicles (EVs) [7]. These vesicles can carry various sorts of signals, including protein factors and miRNA. A comparison of the mouse uterine fluids prior to and during diapause indicated that numerous exosomes are present and enriched with nucleic acids (Bisogno et al., abstract only). Further analysis indicates that the number of these vesicles increased as diapause ensued. This exciting preliminary information indicated that extracellular vesicles may play a role in maintaining and/or terminating diapause.

\section{Future directions}

It was encouraging to observe that many young scientists that have begun investigation of the fascinating domain of embryonic diapause. There is much yet to learn about the phenomenon in the large number of species that display this trait. Although there was some information about seals, wolverines and pandas, the focus of the meeting and these proceedings is predominately on 
the three best studied species, the mouse, the mink and the tammar wallaby. In contrast, we know very little about some, such as the armadillo. Comizzoli (this volume) describes some of the current unknowns, from which perhaps the most significant remains the lack of understanding the dialogue between the endometrium and the embryo that initiates and terminates diapause. Hildebrandt et al. (Wauters et al., this volume, and abstract only) asked whether diapause could be useful for conservation for transfer of in vitro derived embryos. As noted above, global approach initiatives have been taken to establish the uterine signaling and the embryonic response [8] but, for the most part, candidate gene studies (for example MSX, polyamines, mTOR, $c M y c$ and LIF) predominate. Exploration of the role of miRNAs such as Let-7 in EVs [9]3] seems to be a fertile avenue for further investigation.

\section{Conclusions}

The overall goal of the Third International Symposium on Embryonic Diapause was to bring together investigators in this field, and, to update of research progress into the understanding of the enigma of embryonic diapause. The parameters of investigation to be considered were wide, with the vision of integrating new organismal, cellular and molecular findings, as well as to highlight research from the young scientists who have contributed to unravelling the mysteries or diapause. The success of the Symposium in addressing these aims exceeded the expectations of the organizers, as attested to by the high-quality manuscripts that comprise this volume.

The major advances in understanding embryonic diapause reported at this meeting militate for more frequent convocations of international symposia on this phenomenon. Indeed, much of the new information that ensued from the Third International Symposium was fueled by advances in technology that permitted avenues of exploration previously unavailable. The next symposium should be held within the next five years, and it is more than reasonable to predict that it will yield a similar gain in understanding of the enigma of embryonic diapause.

\section{References}

[1] Kamemizu C and Fujimori T. 2019 Distinct dormancy progression depending on embryonic regions during mouse embryonic diapause. Biol. Reprod. 100:1204-1224.

[2] Cheong AW, et al. 2014 MicroRNA Let-7a and dicer are important in the activation and implantation of delayed implanting mouse embryos. Human Reproduction 29:750-762.

[3] Stewart CL, Kaspar P, Brunet L, Bhatt H, Gadi I, Köntgen F and Abbondanzo SJ. 1992 Blastocyst implantation depends on maternal expression of leukaemia inhibitory factor. Nature 359(6390):76-9

[4] Van der Weijden VA, Bick JT, Bauersachs S, Arnold GJ, Fröhlich T, Drews B and Ulbrich SE. 2019 Uterine fluid proteome changes during diapause and resumption of embryo development in roe deer (Capreolus capreolus). Reproduction 158:13-2

[5] Fu Z, Wang B, Wang S, Wu W, Wang Q, Chen Y, Kong S, Lu J, Tang Z, Ran H, Tu Z, He B, Zhang S, Chen Q, Jin W, Duan E, Wang H, Wang YL, Li L, Wang F, Gao S and Wang H. 2014 Integral proteomic analysis of blastocysts reveals key molecular machinery governing embryonic diapause and reactivation for implantation in mice. Biol. Reprod. 90: 1-11

[6] Martin FC, Ang CS, Gardner DK, Renfree MB and Shaw G. 2016 Uterine flushing proteome of the tammar wallaby after reactivation from diapause. Reproduction 152: 491-505.

[7] Kurian NK and Modi D. 2019 Extracellular vesicle mediated embryo-endometrial cross talk during implantation and in pregnancy. J Assist Reprod Genet.36:189-198. 
241

[8] Hussein AM, Wang Y, Mathieu J, Margaretha L, Song C, Jones DC, Cavanaugh C, Miklas JW, Mahen E, Showalter MR, Ruzzo WL, Fiehn O, Ware CB, Blau CA and Ruohola-Baker H. 2020 Metabolic Control over mTOR-Dependent Diapause-like State. Dev Cell. 52:236-25

[9] Deng L, Li C, Chen L, Liu Y, Hou R and Zhou X. 2018 Research advances on embryonic diapause in mammals. Anim Reprod Sci. 198:1-10. 